\title{
Alfred Gräfe †
}

Imuier kleinei · wird die Zahi jeuer Hitter void Geist, die miter Albreeht vou Gräfe1* Führung thatkräftigen Antheil an dem Auf-bau der neueren Augenheilkunde nelimen di $\lceil\cdot f \dagger e n$. den die Erfindung rles Augenspiegels durch Hermann von II elm hoi tz urn die Mitte imseres Jahrhunderts ermöglichte. Aim ist aucli Alfred Gräfe dalúngegangen, der begabtesten , schaffensfreudigsten und fruchtbarsten Schüler des grosseti Meisters einer!

(u)' selten vereinigt die Xatur in gleicli harmouiseher Weise Geist Gemüth, Charakter und fascinirende Persönlichkeit, um das Vor-bild eines wahren Arztes zu schaffen, wïe dies bei Alfred Gräfe der Fall war. Hochbegabt, von gliihender Liebe zur Wissenschaft beseelt und rastlos bemüht, sie stetig zu fürderi, stellte or sieli selbstlos und ganz in den Dienst der leidenden Menschheit, weit, weit iiber das Mass seiner Kräfte liinaus. Es war erhebend und zugleich anspornend, die unversiegbare , gleichmässige Liebenswürdigkeit, die wahre Menschenfreuiidliclikeit und Uerzensgüte auch danu bewundern zu können, wenn die Fülle der praktisclien Thätigkei† ilm zu erdrüeken drolite. Geradezu rührend gestaltete sicb dalier oftmals aucli die Anhänglichkeit, Hochacli-tung, Verehrung und Liebe seiner Krankeu. die aus alien Gesellschafts-kreisen nicht nur der sächsischen L-ande, sondern des gesammte $\alpha$ Dentsclilands, ja man kann vielleicht sagen Europas in der Hallenser Ivlinik zusainmen strömten! Am 23. November 18oü auf Schloss Martinskirchen bei Mühl-berg a. d. E. geboren, besuchte Alfred Gräfe das Realgymnasium der Franke"schen Stiftungen zu Halle a. S. Nach AbsoMrung des-selben bezog er, urn Medicin zu studiren, znerst die $\Gamma$ niversität 1·Jeidel-berg, liieranf die Ilo $<$ discliulen Wiirzburg, Halle, Leipzig, Prag, Berlin. Kin Aufenthalt in Paris bildete den Abschluss der S†udien. Seine ausgesprocliene niatlieniatisclie Yeraulagung, uocli mehr aber der gewaltige Rinfluss seines Vetters Albreeht von Gräfe bestimmten ihn. sich nach Ablegung der Examina in Halle speciell der Augenheilkunde zu widmen. Zu dem Zwecke trat er 1853 als Assistent in die Klinik des Letzteren ein und verblieb in dieser Stellung bis 1858. Der stete, anregende ümgang, sowie das innige Freundschaftsverliältiiiss zu dem genialen Albreeht wirkten mächtig auf sein empfängiiches Gemíith ein. Mit wahrer Be-geisterung vertiefte er sich in die Wissenschaft und legte so den sicheren Grund fiir die spätere eigene Thätigkeit. 1858 wandte sich Gräfe oachHalle zurück und habilitirte sich hier fiir dasFach der Augenheilkunde.

Gleichzeitig errichtete er eine Augenklinik, vorerst von 9 Krankenbetten, die schnell zu ausserordentlichem Ansehen and -grosser Ausdelmung gelangte. 1864 erfolgte die Ernennung zum ausserodentlichen, 1873 diejenige zum ordentlichen Professor. Ende der achtziger ,Tahre machten sich die Folgen der Ceberanstrengung durch die Riesenpraxis bemerkbar. Gräfe war gezwungeu seine Thätigkeit einzuschränken, konnte dadurch aber die alte Elasticität nicht wieder erlangen. Noch einmal, zur Zeit der Xaturforscherversammlung zu Halle, wollte es scheinen, als sei die frühere Kraft zuriickgekehrt. Indessen diese 628

Alfred $\mathrm{Gr}^{7} / 8 £$ £ $\uparrow$.

Hoffnung erwi.es sicli als trügerisch. So salt er sich denn genöthigt, 1892 nicht nur das I, ehraiut und die Direktion der neuerbauteii. schönen Augenklinik niederzulegen. sondern auch auf 
jegliche ärztliche Wirksamkeit zu verzichten. LTm die Ruhe ga,tiz geniessen zu köimen. siedelte er $1 S_{\mid}^{\prime}$ tti nach dem scliöueti Weimar über, sicli ausschliesslich wissenschaftlichen und schriftstellerischen Besehäftigungen widmend.

Gross miissen die 1.1/8folge genaunt werden, die Alfred Gräfe als wissenschaftlicher Autor, Lehrer, gliickliclier Operateur und genialer Arzt errang.

Abgeseheu von der trefflichen Schule, die aus der Reihe der latigjährigen Assistenten erwuchs, ich nenne nur Bunge, Schreiber, Braunschweig, ist auf die grosse Zahl in- und ausländischer Ophthal-mologen hinzuweisen, die fast ständig zur Vervollständigung ihrer Aus-bildung in der Hallenser Klinik weilten. I'nd in der That, der feine klinische Takt, mit dem in complicirten Fallen die Diagnose en $†$-wickelt und durch Mittheilung seltener analoger Beobachtungen gewürzt wurde, gab item Uuterriclite Alfred Gräfe's ein besonderes Piedestal.

Zahlreich and hervorrageud sind die $\Lambda$ vissenscliaftliclien Leistuugen. Dieselben bewegen sicli vorwiegend auf dem Gebiete der klinischen Augenheilkunde, deren Pílege Alfred Gräfe um so mehr am Herzen lag, als er glaubte. dass ílir $\alpha i c h t$ überall die gebülirend $<$ i Be-achtmig gesehenkt werde. Es $\Lambda$ vürde zu weit füliren, wvllten wir aucl·i uur die Titel aller Arbeiten und Mittlieiluugen aufzählen. die seiner Feder entstammten und die ein endgiiltiges Zeugniss der Viel-seitigkeit nicht minder wie der ausserordentlichen Gründlichkeit und Klarheit ablegen. [Teberall, aucli in dej · unschembarsten Notiz, ñnden wir einen klinischen Gt;da.nken zuin Ausdruck gebracht und meist durcli werthvolle Beobachtungen gestützt. Auf drei Gruppen von Arbeiten möchte ich aber die Aufmerksamkeit lenken, da sie, man kann wohl sagen die Lieblingsthemata Gräfe's behandeln.

Schon während der Berliner Assisten†enzeit entstand die »ruhd-legende Monographie Kliuische Analyse der Motilitätsstörungen des Auges" (1858), welche dieses so wichtige Kapitel gewissermassen erst erschloss. Dem weiteren $\Lambda$ usbau derselben widmete der Autor die ungetheil-teste Aufmerksamkeit bis an das Jjebensen.de. $\lambda$ 'oeh zweimal unter-warf er sie einer systematischen und vervvllständigenden Bearbeitung, einmal in dem ílandbuche der gesammten Augenheilkunde, welches er gemeinsam mit Th. Sämisch von 1874 an edirte, das andere Mai. in der Xeuauflage eben dieses grossen Werkes am 1/1/8de des verflossenen Jahres. Der Tod überraschte ihn noch vor der endgültigen Fertig-stellung. Die letzte Lieferung konnte, wie ich vernehme, nur im Manuskript vollendet werden.

Ausser diesem Cyclus von Arbeiten. zu dem auch die aus-gezeichnete Abhandlung über das Sehen der Schiele $\pi$ den (18 97) gehört, werden Gräfe's Forschungen und Vorschläge zur Entfernung von Cysticerken aus den tieferen und tiefsten Theilen des Bulbus mittelst meridionalen Scleralschnittes einen dauernden Platz in der Literatur †inde $\tau$. Denn es wurde durch dieselben nicht nur die Extraction des Blasenwurmes in den meisten Fallen überhaupt erst ermöglicht.

iagesnaeliricht.

629

aucJi mannigfache andere Eingriffe im liinteren Augenabschnitte basiren auf der allgemein anerkannten Operatioasmethode.

Fernerhin erwarb sieli Alfred Gräfe unseres Erachtens bleibende Verdienste urn die operative Augenheilkunde durcli die Einführung der antiseptis $<\cdot$ hen Cautelen. N'ach jahrelangen Versuchen gelang es ihm, eine Anwendungsweise der Lister'schen Antisepsis für das Sehorgan zu finden, welche das $>$ Schreckgespenst der Suppurationen, zumal nach der Staarausziehung aus,schaltete oder doch zu den grössten Séltéti-heiten (in kaum 1 pCt. der Fälle) stempelte. 
Tausende und Aber-tausende von Auge $\pi$ werden durch dieselben seitdem jährlich in ihrem 1> esfande erhalten - znm danernden Rubme Lister's und Alfred Gräfe's!

So sehr Alfred Gräfe durcli die übergrosse klinische and practische Thätigkeit auch in Anspruch genommen wurde, er fand immer nocL - Zeit, urn sich der Kunst und schöngeistigen Neigungen hinzugeben. In ihnen suchte und fand er die nöthige Erholung, aus ihnen schöpfte er gewissermassen ne $\pi$ e Kraft für den Beruf und den geistigen Aufsehwung.

Sichern die wissenschaftlichen Thaten Alfred Gräfe für immer einen Platz in der Geschichte der Ophthalmologie, so werden uicht minder seine hohen persönlichen Tugenden, sein lauterer Charakter. seine Offenbeit und Gradheit, gepaart mit grösster Liebens-wiirdigkeit, seine Freundschaft und Treue bei alien, die ihm persönlicb uäher treten durfteu, das Gefühl unauslöschlicher Verehrung und Dankbarkeit wacli erhalten. Der harte Kampf im Leben bewahrt nur wenige Sterbliche vor Feinden, Alfred Gräfe gehürte zu diesen wenigen. Seine vornehme Gesinnung und .sein uneri $3 / 8$ chütterlicher Gerechtígkeitssinn, die alles Kleinliche weit abhielten, nicht zuletzt aucli seine ganz u $\pi$-gevvöhnliche Bescheidenbeit, Milde und Güte konnten wohl Neider, aber keine eigentlichen $\lambda$ Vidersaeher gross werden las, sen.

Man wird in der Polgezeit vielleicht schwanken können, ob manan dt·iu Dahingegangeuen melir den hochverdienten Gelehrten, dengenialen Operateur oder den bedeutenden und gliicklichen Arzt bewundernsoil, in keinem Fade aber wird ihm die Anerkennung versagt bleiben:Er war ein Mann! Kuhnt.

Tagesnachricht.

D. Die neuerbaute Üniversitäts-Augeuklinik zu Breslau wurde am I. April cr. feierlich direr Bestiøimung übergeben. Der Director der-selben, Prof. üv. libthoff, würdigte in eingehender Rede die Ent-wicklung der modernen Augenheilkun.de, insbeso $\pi$ dere der letzen Jahrzehnte und wies auf die Ziele, namentlicb der aetiologischen For-schungen bin. 